\title{
Piece of Cake: Assuring Specific Qualities of Product in Farm Lifecycles with DLT - Can Evidenced-Based Practice be supported by Participatory Action Research Methods?
}

Hannah Rudman

SRUC, Scotland's Rural College, UK

Correspondence: hannah.rudman@sruc.ac.uk

Received: 24th September 2020 Accepted: 16th October 2020 Published: 27th October 2020

\begin{abstract}
:
A strong need for evidence-based practice in the blockchain and distributed ledger technology (DLT) research, development and action domains is currently clarifying. Literature highlights a lack of transparency around the outputs, outcomes and impacts of blockchain projects. As previously cited in an article of this journal, for example, the US Agency for International Development studied 43 projects and found that nearly all 43 did not want to share their results [1]. The Centre for Evidence-Based Blockchain recently completed a study of 517 companies to see if their blockchain projects could be defined as evidence-based practice. Over four years they measured companies using the PCIO framework (what evidence is there of Problem - Comparison - Intervention and Outcomes) of evidencebased practice. The studies concluded that almost half of the blockchain companies showed 'no explicit evidence of the problem to be solved. Approximately one-third fail[ed] to cite a comparison and intervention analysis, and less than $2 \%$ demonstrate[d] evidence of outcomes backed by filtered (critically appraised, peer reviewed) information’ (Naqvi \& Hussein, p. 8 [2].)

This article presents how qualitative research design and methodologies can help companies and academics achieve evidence-based practice. It presents a case study, in the PCIO framework, of a small-scale agriculture sector project to assure a specific quality. The case study is a conclusion of a project that was run as participatory action research (PAR), involving a consortium including academics, farmer practitioners and a technical DLT platform developer, between 2018 and 2020. The findings show that PAR is an appropriate research method for any democratic collaborative consortia to achieve evidence-based practice through dialogue, discussion, codevelopment and trusting relationships.
\end{abstract}

Keywords: distributed ledger technologies (DLT), participatory action research (PAR), case studies, agri-food supply chains, research and development design, qualitative research methodologies, evidence-based practice.

JEL Classifications: O3, L6, and Q1.

\section{Introduction}

There are high-profile agri-food sector blockchain case studies from the biggest sector companies working with tech giants such as IBM's Food Trust (its website features seven case studies, with IBM's blockchain solution improving supply chain efficiency, food safety, waste and fraud, brand trust, etc.) [3]. Their purpose is to mainly serve as marketing tools, but the case studies do report evidence of problems solved by the IBM solution. Both regulatory direction and consumer demand are pushing blockchain technology into agri-food supply chains. The U.S. Food and Drug Administration (FDA)'s 2019 initiative The New Era of Smarter Food Safety [4] was built on the 2011 Food Safety Modernization Act (FSMA) by suggesting a modern approach to food traceability. This accelerated a number of blockchain proof-of-concept projects. FoodLogiQ, IBM Food Trust, ripe.io and SAP simulated seafood supply chain data sharing by leveraging GS1 standards, the most widely used supply chain standards in the world. Blockchain technologies in the project facilitated more accountability in the supply chain, through multiple parties across a supply chain supplying data forming an immutable ledger or audit trail of product events and transactions [5]. In August 2020, the U.S. Department of Agriculture said it envisioned distributed ledger technology (DLT) becoming integral to the functioning of complex agricultural supply chains in the future [6]. Chinese consumers became even more interested in transparency during the COVID-19 crisis. In response, the APAC Provenance Council was formed in 2020, including VeChain and Blockchain Australia, again leveraging GS1 standards. By combining resources from all members, the Council aims to provide a comprehensive blockchain-enabled food supply chain finance ecosystem, bridging traceable, safe and trusted trades with shorter billing terms between Australian suppliers and Chinese importers, as well as proving traceability of product [7]. Global-scale hi-tech food supply 
chain companies are progressing the development of national and international traceability pilot projects with solutions that include blockchain technologies to provide transparency and traceability, as well as improve the speed of tradability [8], [9]. This all builds on Opara's vision from 2002, discussing the future prospects for traceability in the food supply chain, and correctly predicting that access to better hardware and software would eventually enable 'the development of electronic identification (EID) systems, which include electronic tags with chips and handheld scanners for reading, storing and transmitting the data to PCs for analysis and longterm storage' [10].

Food supply chains, whether agri- or aqua- focussed, are conceptually similar, and work as a linear chain of custody of different actors. All food supply chains start with a grower/producer - the producer might be nature itself, or a farmer working with natural resources. Distributors (hauliers) then take over the chain of custody of the farmed and harvested product when they transport it to food processors, where natural products are then either blended or divided into packaging or combined with other ingredients. The processor sells the end product to retailers who in turn sell to consumers. Drawing on agriculture and food supply chain literature (e.g. [11], [12]), Parmar and Shah review a number of past and current blockchain projects and suggest a series of issues suffered by each of those actors along the chain that could be improved by the application of blockchain and other technologies (pg. 5926, [13]). Before them in 2015, the Provenance Project recognised the value of consumers and the chain of custody actors in food supply chains by providing them with documentation about a product's origin and journey through the supply chain via a trustable data format in their whitepaper [14]. The paper initially suggested a decentralised application (Dapp) based on the Ethereum blockchain to be the trustable data source. Provenance has since developed a transparency platform and consultancy business, and has worked with the global food brand Princes Group, to provide blockchain tracking and verification for fish and fruit supply chains; with the International Pole and Line Foundation for fish; with Marleybones for pet food; and Bridgehead for coffee (all case studies can be read at provenance.org). Systematic literature reviews of blockchain technology in agriculture mainly discuss the countries where the most activity in the sector is happening - China is the leader with most academic publications about agriculture sector blockchain projects, followed by USA, Italy, India and Spain [15]. The academic literature reveals trends, with research focussed on traceability, security design and blockchain networks as information systems [16]. However, there are far fewer small-scale projects discussed in the literature that focus on the collection of data about what happens to produce on farms, when it is in the chain of custody with the grower/producer. This is just as important as some consumers need to be assured of specific qualities being constantly present in products throughout the entire lifecycle due to health challenges.

This article focusses on a small-scale DLT in agriculture project, showing what evidence there is of Problem - Comparison Intervention and Outcomes, a framework recognised by Naqvi
\& Hussein [2] for proving evidence-based practice. The project was a social research involving interdisciplinary collaboration, across a range of disciplinary and organisational boundaries. But what does this mean for research practice? How important is participatory action, connectivity and collaboration in research design? Participatory action research (PAR) is a broad term covering a range of participatory approaches to actionorientated research. It has great practical value in interdisciplinary research practice, common when working with external partners for collaborative project outcomes. PAR involves researchers and participants working together to actively investigate a problematic situation or action in order to change or improve it for good [17]. This article shows that PAR is an approach for academic researchers and external organisations to work together, to co-produce meaningful research designs and practical collaborative project outcomes, as well as prove evidence-based practice.

\section{Principles of PAR}

The principles of PAR originated over 70 years ago with Lewin and the Tavistock Institute [18]. It is practice-led, rather than practice-based, and contrasts with traditional scientific research where participants are objects of the study. The PAR methodology is structured as a 'cyclical process of fact finding, action, reflection, leading to further inquiry and action for change' (Minkler, p. 191 [19]). The approach includes collective fact-finding, analysis and decision-making involving egalitarian participation by a team, community or organisation to transform some aspects of its situation or structures through action, research and experience (p.1 Reason \& Bradbury [20], [21]). As such PAR practitioners attempt to integrate three aspects: participation (life in society and democracy), action (engagement with experience and history) and research (soundness in thought and the growth of knowledge - Chevalier and Buckles, pp.6-8 [22]) with practical actions seamlessly uniting with research (Chambers, p. 315 [23]) and typically being performed 'with' people and not 'on' or 'for' people (Chevalier and Buckles, p. 5, [24]). PAR provides a genuine co-learning process through which different ways of knowing are valued and integrated and importantly the research process is considered to be as significant as the outcome (Pain and Francis [25]).

The PAR approach typically helps to create actionable knowledge, or interventions, for organisations facing difficulty and change by reflecting on and learning from the organisation's reflections and learning, respectively. It is this idea of meta-learning through the inclusion of academic and practitioner reflection that elevates action research above everyday problem solving [26], [27]. PAR can be particularly effective for multidisciplinary research. PAR approaches focus on enabling full participation of all those involved in the research process [28], and forging partnerships so participants can explore possibilities for transformation together [29]. Although collaboration has become common within the social sciences, there is evidence that multidisciplinarity is only now becoming more accepted and understood in the wider academy [30]. 
PAR is an approach based on a set of core values that follow a broad process, rather than specific methods mapped out in advance. Together, project teams work iteratively to develop the focus of interest, methods and findings, sometimes dividing up tasks according to experience, and always reflecting at each stage. Both the enquiry and decision-making are therefore open and jointly negotiated (see Pain, Kesby and Askins, [31]). This involves the creation of a culture of systematic reflection within the project team. In order to create this culture of reflection it is important to be as open and transparent as possible and to actively include all stakeholders, and the project team, in the research design process. While this might at first appear to be at odds with the usual systematic research process, it has been suggested that it does not fundamentally alter the research method: rather, it places it within a process where it is developed and discussed by a group that has a range of perspectives, knowledge and expertise [32].

This participatory action research consortium involved SRUC - Scotland's Rural College, a collective of farms, and DLT platform software developers working collaboratively to cocreate research and action in the form of a proof of concept technology demonstrator.

\section{PCIO case study}

PROBLEM: This project originated from an enquiry to SRUC Scotland's Rural College - from a group of farms in the north east of Scotland in 2018. They sought a reliable method of providing traceability, provenance and assurance of the glutenfree oat crop that they grow. Although oats are naturally gluten free, some manufacturers require assurance that they are not contaminated with other grains that may contain gluten. Some consumers need to be assured of specific qualities in food due to health challenges. For example, auto-immune response in people with Coeliac disease or severe gluten allergies $(1-2 \%$ of most populations) is triggered when consuming more than 10 $50 \mathrm{mg}$. Most health authorities define gluten-free products as containing less than 20 parts per million gluten [33]. Oats are naturally gluten free, but can become contaminated (e.g. by wheat, barley or rye) as they grow and are harvested and stored on the farm, or are processed or transported by food manufacturers. This contamination risk makes them an unreliable food source for Coeliac disease sufferers. Although there is no official gluten-free assurance scheme, the farms have developed their own protocols to ensure that no contamination takes place on the farms, and required a mechanism to provide details and proof of this to the rest of the supply chain. Understanding the capabilities of blockchain technology for agriculture, SRUC held initial meetings with a DLT platform technical development company, to see if the farms' requirements could be met by the DLT platform. As a consortium, we developed a participatory action research and technical development approach.

INTERVENTION: Funding was secured for a project from The Scottish Government's Rural Payments and
Services Department's Knowledge Transfer and Innovation Fund (2019). A DLT platform was used to establish a decentralised private network between the farmers, SRUC (acting as verifiers) and third-party validators to enable them to co-create a persistent digital record through time of data transactions about the oats. All parties in the network stored the decentralised record for resilient information security. The DLT platform also enabled the collaborative building of a shared but permissioned and encrypted digital register, which collected and secured data about the oats' GF status, throughout their growth lifecycle from the different participants. We brainstormed and mapped this process using Visio as a tool to construct a diagram, defining what actor undertook which step, and what digital data were required to prove its validity. Some process steps required sub-steps, where a number of processes would be repeated in the parent step through time (Figure 1).

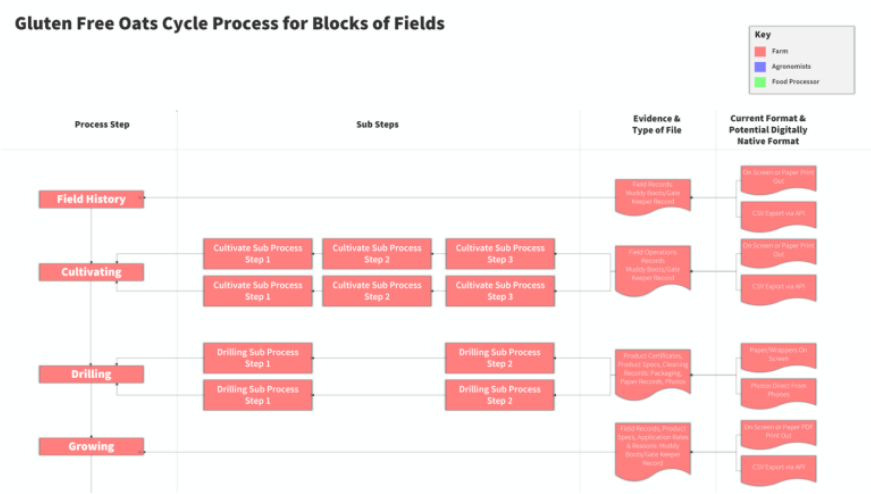

Figure 1: The process mapping of steps and data required as proof in the gluten-free oats cycle.

We then constructed it as a process of steps by actors in the DLT platform's process designer user interface (Figure 2).

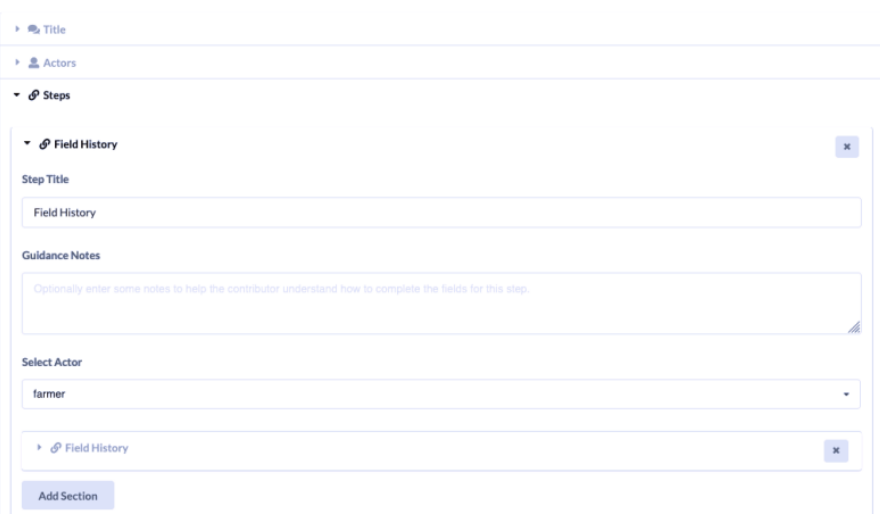

Figure 2: The SICCAR DLT platform's process design user interface (see http://wallet.services).

The farmers and verifiers in the private network could both read from and write on the register via a controlled process in a programmatically governed way. Each party only had access to write or read the data for which they had explicit 
permissions. Permissioning was agreed by all the parties via a function in the process designer user interface, and was written as cryptographic rules to the shared register as part of publishing a multi-party process (Figure 3).

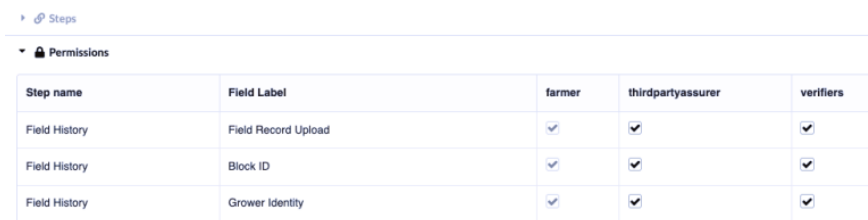

Figure 3: Granular permissioning of each piece of data in SICCAR (see http://wallet.services).

Data was only decrypted if a participant is a member of the wallet that the data transaction was sent to (proof of authority is the consensus mechanism the DLT platform uses). Figure 3 shows that the Field Record Upload, the Block ID and the Grower Identity data, all part of the Filed History step in the process, should be decrypted by the Farmer, Third-Party Assurer and the Verifier actors. Access to the actors' wallets is controlled by adding and removing delegates from wallets, and this was managed using each organisation's pre-existing enterprise user authentication and ID management system or directory, and the user management application in the DLT platform. Webforms gave access to all actors in the network through a simple web address, where they could only see actions and data relevant to them, minimising compliance and regulatory obligations. (Figure 4 shows the Farmer's first actions in the shared process, requiring data upload via a webform.)

\section{Field History

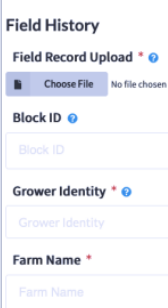

Figure 4: Webform viewable by the Farmer actor, requiring data upload into the DLT platform (see http://wallet.services).

Farmers' data had to be validated as being true by the verifiers. Third-party assurers were given access to certain data to audit for certification. Once written on to the register, the data was encrypted so it could not be tampered with. The DLT platform's directed acyclic graph (DAG) architecture model enabled the representation of complex split and combined chains, and for agricultural processes that at points had multiple repetitive steps - Figure 1 shows the requirement for the cultivating step to have a number of sub-steps.
API access to the public data, provided for transparency on the register in unencrypted format, powered a mobilefriendly web app that consumers could access via their phone's camera capturing a QR code to trace and track the gluten-free status of the oats using the data defined as public (Figure 5).

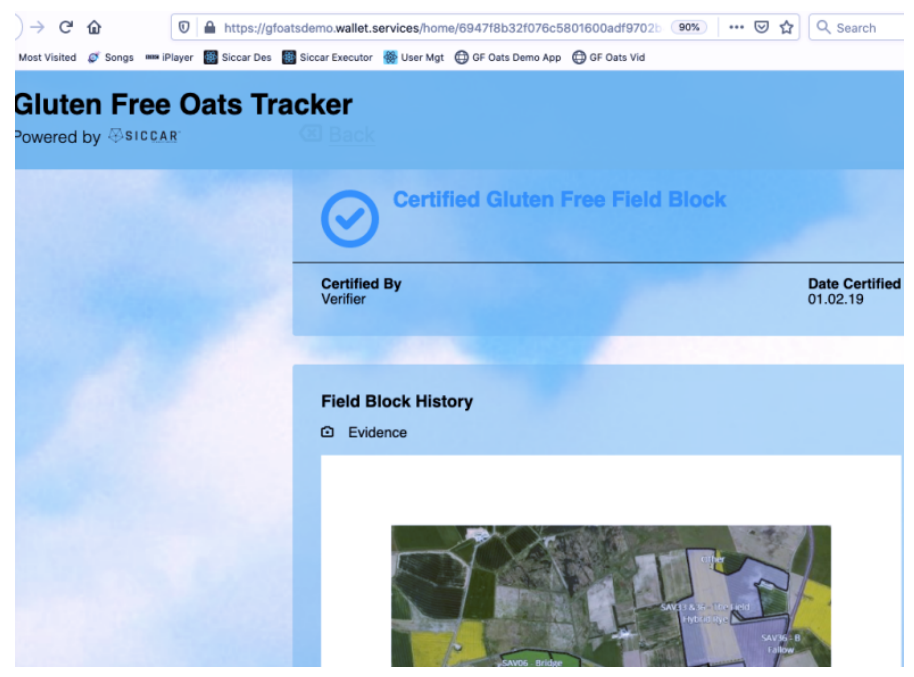

Figure 5: Unencrypted data defined as public, and so viewable to the API, and powering a mobile-friendly web app for consumers to view.

This app was launched by consumers taking pictures of a QR code on the packaging of the oats (Figure 6).

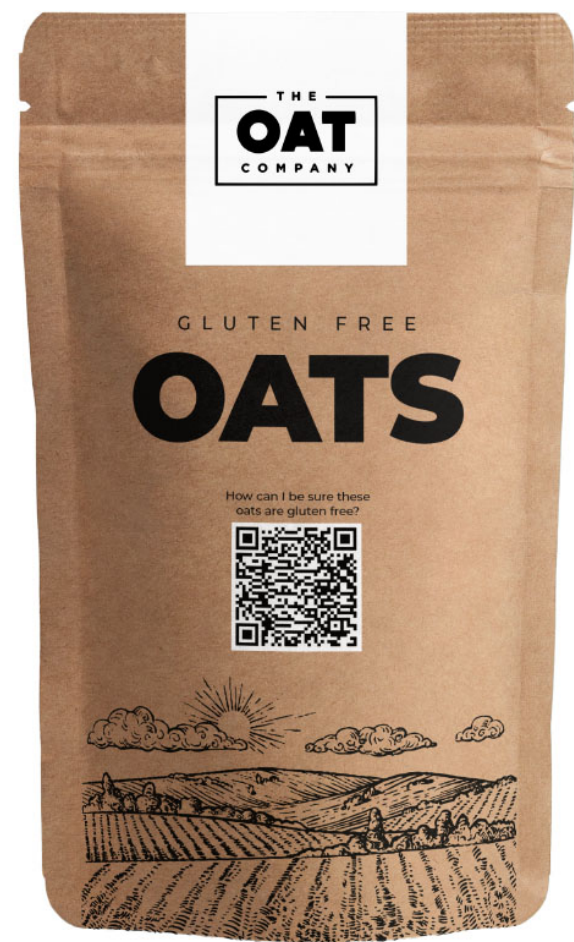

Figure 6: The QR code on the packaging of the oats, which opens the app showing public data, to prove their verified and assured gluten-free status. 
The output of the project was the construction of the secure, private, permissioned DLT network and the publishing of a shared rules-based process on an encrypted distributed register, which was accessible to the consortium members through a user interface of easy-to-access mobile-friendly webforms governed by wallet services. This live and tangible output - a proof of concept (POC) - had a number of outcomes.

OUTCOMES: The project's outcomes, a live, published POC that could be interacted with and demonstrated, proved that the DLT solution enabled and facilitated the provision of transparency to consumers (see Figures 5 and 6, above). Consumers could track provenance, and trace and monitor gluten-free levels of the oats throughout their lifecycle from seed to shop. It also tilted some power in the supply chain back to the farmers, as they were able to evidence the quality of their processes to buyers and food producers for a better price. This builds on the hypothesis that value distribution becomes fairer with increasing transparency as proposed by Gardner et al [34]. In our case, the DLT solution was an economically fairer sociotechnical development for farmers. The DLT platform developers received a license fee, and SRUC had a live POC, which could be demonstrated to achieve impact in the sector: 100 professionals in the sector experienced demonstrations at the Future Farming workshop in Aberdeenshire on 19 February 2020. There have been another 100 views of the YouTube video demo of the POC [35]. The live and video proof of concept demonstrations, and short online qualitative case study report further attracted the interest of the press and generated stories. In September 2020, there had been one international BBC programme produced that featured the project and its case study [36], one national press story ([37]), two regional press stories ([38], [39]), and four sector press stories ([40], [41], [42], [43]) - significant external coverage, although actual reader numbers cannot be measured from these external sources.

\section{Discussion}

Knowledge transfer to the agricultural sector was a key impact enabled from publishing the participatory action research project as a PCIO case study, which the press picked up on. Another impact of utilising PAR during the lifecycle of the project meant we were focussed on generating outcomes for all parties' benefits. PAR also demands reflection and evaluation, at the end of cycles, and a summary of learnings within them. For the DLT platform developers, there were learnings that became part of their platform through their agile software development processes: the idea of steps and child steps. This was needed for agricultural processes that had multiple repetitive steps (see Figure 1) and for processes that needed to eventually combine. Halfway through a harvest cycle, adding data to a register, the consortium recognised the need to consider what would happen if a field or harvest failed to be gluten-free due to contamination, therefore requiring the ending of a register (the oats continue into the supply chain for food production, but without the special quality being guaranteed). The design of the register therefore changed at mid-point, and at the end of the harvest, when it was recognised that registers tracking field blocks needed to combine to become the single record for the store.

The learning for SRUC as validators of evidence was that sometimes the best digital evidence would be pictorial and direct from a users' smart device in their pocket on the farm. The metadata of the picture provided the triangulation data proving date and time of pictorial evidence and location of device. The learning for the farmers was that any data they input into the new DLT system ended up being an irritating time-wasting duplication of effort, and that for the system to be an acceptable IT addition, data input would need to be automated from edge devices such as sensors, and that Bring your own Device would need to be strongly authenticated securely into the DLT network.

\section{Conclusions and further research}

Participatory action, connectivity and collaboration were important in our applied research and development project. The consortium agreed that the PAR project resulted in a proof of concept which proved the technical viability of DLT, and as a case study in the PCIO format, this gained sector and press interest. The need to automate evidence directly from machines - hardwares such as IoT devices and softwares such as sector-specific management systems - as well as from user devices not necessarily in wallets is a technical challenge to overcome next. All hardwares and softwares would need to be strongly authenticated and validated to be acceptable into a secure, private and permissioned DLT network as an actor. The business model was not proven by the project or the PoC, and this would also need to be worked out as part of a more extensive pilot and roll-out.

PAR as an action-focussed cyclical process enabled the consortium's project and fitted with natural cycles of growth and harvest, as well as agile software development cycles - it presented a very democratic mode of approaching research, learning and the action of technical development.

Multidisciplinary collaboration with external partners enables a type of radical knowledge co-production that can enhance the learning, knowledge and expertise of all those involved, leading to positive research outcomes. Despite the potential benefits of the PAR approach when working with external partners on multidisciplinary collaborative projects, there are a number of organisational barriers to be considered. However, PAR provided the framework to establish research questions, develop methods, conduct collaborative data collection and analysis and produce outputs, but the details of the process must be context-specific. This has meant that to date, PAR and co-production projects occur at a relatively small scale [44]. As agri-food-focussed DLT proof of concept projects 
and pilots continue and mature, processes will be longer and more complex as different parts of the supply chain join in. The democratic and collaborative nature of bigger, longerstanding DLT networks will find that PAR is an appropriate research method to achieve evidence-based practice and provable outcomes and impact through its focus on dialogue, discussion, co-development and trusting relationships.

\section{Competing interests:}

HR worked as an employee of the DLT platform provider used during the lifetime of the PAR project, and was a member of the democratic team, collaborating in the PAR process. The PCIO case study was written up, as was this article, once she joined SRUC as an employee in August 2020. SRUC has no special interest in the DLT platform: applied research and development projects across the organisation use a number of different DLT platforms.

\section{Ethical approval:}

All actors in the $P A R$ research granted their permission to be mentioned as actors in any public case study.

\section{Author's contribution:}

HR designed and coordinated this PAR project, developed the PCIO case study and prepared the manuscript in entirety, with the exception in the acknowledgement below.

\section{Funding:}

The PAR project was funded by Scottish Government's Rural Payments and Services

Department's Knowledge Transfer and Innovation Fund 2019.

\section{Acknowledgements:}

HR would like to thank Dr Claire Bailey-Ross from the University of Portsmouth UK, Dr Jeremy Kendal, Dr Zarja Mursic and Dr Rachel Kendal all from the Durbam University UK; Andy Lloyd of the Centre for Life, Newcastle-upon-Tyne; and Bethan Ross of the Science Museum, London for the work on various $P A R$ focussed papers which contributed to the Principles of the PAR section of this article. HR would also like to thank W allet Services for agreeing to share the DLT platform's images in the figures.

\section{References}

[1] J. Burg, C. Murphy, and J. P. Pétraud, "Blockchain for International Development:

Using a Learning Agenda to Address Knowledge Gaps," 29th Nov 2018,

[2] N. Naqvi and M. Hussain, "Evidence-Based Blockchain: Findings from a Global Study of Blockchain Projects and Start-up Companies," The Journal of the British Blockechain Association, vol. 3, no. 2, 2020, doi: https://doi.org/10.31585/jbba-3-2(8) 2020 .

[3] IBM. "IBM Food Trust case studies." https://www.ibm.com/uk-en/blockchain/ solutions/food-trust (accessed 14th September 2020 .

[4] (2020). 7 CFR Part 205, National Organic Program; Strengthening Organic Enforcement. [Online] Available: https://www.govinfo.gov/content/pkg/FR-2020-0805/pdf/2020-14581.pdf

[5] E. Cosgrove. (2020) SAP, IBM Food Trust, GS1 move toward food supply chain traceability with interoperability test. Supply Chain Dive. Available: https://www.supplychaindive.com/news/gs1-ibmfood-trust-sap-and-more-pass-key-interoperability- milestone-for-f/579631/ (accessed 13th Ocotober 2020).

[6] A. Hajirnis. (2020) The U.S. Department of Agriculture plans to use blockchain to streamline the EKO food supply chain. Blocksats. Available: https://blocksats.com/the-u-s-department-ofagriculture-plans-to-use-blockchain-to-streamline-theeko-food-supply-chain/ (accessed 13th Ocotober 2020).

[7] Blockechain traceability supports brand Australia. [Online] Available: https://minister.awe.gov.au/littleproud /media-releases/blockchain-traceability (accessed 13th Ocotober 2020).

[8] Reshma Kamath, "Food Traceability on Blockchain: Walmart's Pork and Mango Pilots with IBM," The Journal of the British Blockechain Association, vol. 1, no. 1, pp. 1-12, 2018-07-04 2018, doi: 10.31585/jbba-1-1(10) 2018 .

[9] PTI, “Agriota' e-market platform launched to bridge gap between Indian farmers, UAE food industry," in The New Indian Express, 29th August ed, 2020.

[10] L. Opara, "Traceability in agriculture and food supply chain: A review of basic concepts, technological implications, and future prospects," 2002.

[11] M. P. Caro, M. S. Ali, M. Vecchio, and R. Giaffreda, "Blockchain-based traceability in Agri-Food supply chain management: A practical implementation," presented at the IoT Vertical and Topical Summit on Agriculture., Tuscany, Italy., 2018.

[12] A. F. Kamilaris, A. Prenafeta-Boldu', F.X., "The rise of blockchain technology in agriculture and food supply chains," Trends in Food Science \& Technology, vol. 91, pp. 640-652, 2019, doi: https://doi.org/ 10.1016/j.tifs.2019.07.034.

[13] M. S. Parmar, P., "Uplifting Blockchain Technology for Data Provenance in Supply Chain," International Journal of Advanced Science and Technology, vol. 29, pp. 5922-5938, 2020.

[14] Project Provenance Ltd. "Whitepaper." https://www.provenance.org/whitepaper (accessed 13th Ocotober 2020).

[15] V. S. Yadav and A. R. Singh, "A Systematic Literature Review of Blockchain Technology in Agriculture," 2019.

[16] O. Bermeo-Almeida, M. Cardenas-Rodriguez, T. Samaniego-Cobo, E. Ferruzola-Gómez, R. CabezasCabezas, and W. Bazán-Vera, "Blockchain in Agriculture: A Systematic Literature Review," in CITI, 2018.

[17] Y. Wadsworth, "What is Participatory Action Research?," Action Research International, 1998.

[18] K. Lewin, "Action Research and Minority Problems," Journal of Social Issues, no. November, 1946, doi: https://doi.org/10.1111/j.1540-4560.1946.tb02295.x.

[19] (2000). Using Participatory Action Research to build Healthy Communities.

[20] P. Reason and H. Bradbury, Handbook of action research, 2nd ed. London: Sage, 2008. 
[21] D. Coghlan and T. Brannick, Doing action research in your own organisation, 3rd ed. London: Sage, 2010.

[22] J. M. Chevalier and D. J. Buckles, Participatory action research: Theory and methods for engaged inquiry. Abingdon: Routledge, 2013.

[23] R. Chambers, "Chambers, R. (2008) 'PRA, PLA and Pluralism: Practice and Theory.' In The Sage handbook of action research: Participative inquiry and practice. Edited by P. H. BradburyReason. Sage.," in PRA, PLA and Pluralism: Practice and Theory.' In The Sage handbook of action research: Participative inquiry and practice, $\mathrm{H}$. Bradbury and P. Reason Eds.: Sage, 2008.

[24] J. M. Chevalier and D. J. Buckles, A Guide to Collaborative Inquiry and Social Engagement. 2nd ed. New Delhi: Sage.

[25] R. Pain and P. Francis, "Reflections on Participatory Research," Area, vol. 35, no. 1, pp. 46-54, 2003.

[26] D. Schon, The reflective practitioner: How professionals think in action. New York: Basic Books, 1983.

[27] C. Argyris, "Actionable Knowledge," in The Oxford bandbook of organization theory, T. C. Knudsen Tsoukas Ed. Oxford: OUP 2003, pp. 423-52.

[28] R. Shura, R. A. Siders, and D. Dannefer, "Culture change in long-term care: Participatory action research and the role of the resident," The Gerontologist, vol. 51, no. 2, pp. 212-25., 2011, doi: 10.1093 /geront/gnq099

[29] W. Frisby, C. J. Reid, and S. Millar, "Putting "Participatory" Into Participatory Forms of Action Research," Journal of Sport Management, vol. 19, p. Journal of Sport Management, 2005.

[30] H. Lee, "Uncovering The Multidisciplinary Nature Of Technology Management: Journal Citation Network Analysis," Scientometrics, vol. 102, no. 1, pp. 51-75, 2015.

[31] R. Pain, M. Kesby, and K. Askins, "Geographies of impact: Power, participation and potential," Area, vol. 43, no. 2, pp. 183-188, 2011.

[32] S. N. Lane, N. Odoni, C. Landström, S. J. Whatmore, N. Ward, and S. Bradley, "Doing flood risk science differently: An experiment in radical scientific method," Transactions of the Institute of British Geographers, vol. 36, no. 1, pp. 15-36, 2011.

[33] I. S. Cohen, A. S. Day, and R. Shaoul, "Gluten in Celiac Disease-More or Less?," (in eng), Rambam Maimonides Med J, vol. 10, no. 1, 2019, doi: 10.5041/rmmj.10360.

[34] T. A. Gardner and E. al., "Transparency and sustainability in global commodity supply chains," World Development, vol. 121, 2019, doi: https://doi.org/10.1016/j.worlddev.2018.05.025.

[35] Wallet.Services, Demo: Gluten Free Oats assured by SICCAR Smart Registers. Available: https://youtu.be/LoPpD0yxFa0 (accessed 13th Ocotober 2020).

[36] BBC Radio 4, "Oats and Blockchain," in Farming Today, 18th August ed: BBC (International), 2020.
[37] G. Davidson, "Scots farmers pioneer digital 'glutenfree' assurance chain," 11th September ed: The Herald, 2019.

[38] G. Mackenzie, "Simple scan on way to ensure oat provenance," in The Press and Journal, September 4th ed, 2019.

[39] G. Mackenzie, "Technology to trace oats provenance a step closer," in The Courier, 4th September ed, 2020.

[40] V. Bamford. (2020) Gluten-free oat supply chain developed using blockchain technology. British Baker. Available: https://bakeryinfo.co.uk/ingredients / scottish-farms-develop-gluten-free-oat-supplychain/647481.article (accessed 13th Ocotober 2020).

[41] G. Davidson, "Scottish growers aim to guarantee gluten-free oats," The Scottish Farmer, 19th August 2020.

[42] F. U. Team. (2020) Six farmers develop Scotland's first gluten free oat supply chain. Farming $U K$. Available: https://www.farminguk.com/news/sixfarmers-develop-scotland-s-first-gluten-free-oatsupply-chain_56323.html (accessed 13th Ocotober 2020).

[43] G. Selby, "Blockchain boosts food safety and provenance in UK gluten-free oats," Food Ingredients First (International), 24th August 2020,

[44] C. M. Maynard, "How public participation in river management improvements is affected by scale," Area, vol. 45, no. 2, pp. 230-238, 2013. 